\title{
RESIDÊNCIA INTEGRADA EM SAÚDE MENTAL: CUIDADO À REDE DE ATENÇÃO PSICOSSOCIAL
}

\author{
Integrated residency in mental health: care for the psychosocial care network \\ Residencia integrada en salud mental: cuidado con la red de atención psicosocial
}

\author{
Aline Luiza de Paulo Evangelista \\ Escola de Saúde Pública do Ceará - ESP/CE - Fortaleza - CE - Brasil
}

\section{Amanda Cavalcante Frota}

Escola de Saúde Pública do Ceará - ESP/CE - Fortaleza - CE - Brasil

\section{Rafael Bruno Silva Torres}

Faculdade Maurício de Nassau - UNINASSAU - Fortaleza (CE) - Brasil

\section{Ivana Cristina de Holanda Cunha Barreto}

Fundação Oswaldo Cruz - FIOCRUZ/CE - Fortaleza - (CE) - Brasil

Universidade Federal do Ceará - UFC- Fortaleza - (CE) - Brasil

\section{RESUMO}

Objetivo: Compreender o contexto histórico e político-pedagógico da implementação da Residência Integrada em Saúde Mental Coletiva da Escola de Saúde Pública do Ceará (RISMC-ESP/CE) e a percepção dos atores sociais envolvidos na implementação desta sobre os seus reflexos na organização da atenção psicossocial de quatro municípios do Ceará, Brasil. Métodos: Estudo qualitativo descritivo, exploratório e analítico, com análise documental, entrevistas semiestruturadas e análise de conteúdo temática. Resultados: Quatro categorias emergiram, a saber, Contexto histórico e político-pedagógico da implementação da RISMC-ESP/ CE; Educação permanente interprofissional em Saúde Mental (EPISM) nas redes de atenção e no território; Fortalecimento da participação popular e luta antimanicomial; e Desafios da saúde mental. Apesar do subfinanciamento do SUS e das políticas que favorecem o desmonte da Rede de Atenção Psicossocial (RAPS), houve promoção da saúde mental através da EPISM, gerando ações intersetoriais, utilização de tecnologias avançadas de cuidado e estímulo à participação social no território. Os principais desafios são o estigma social da loucura, a consolidação de estratégias de cuidado que superem as instituições asilares e invistam na redução de danos e a estabilidade das equipes de saúde para continuidade das ações construídas. Conclusão: A RISMC-ESP/CE é um dispositivo de resistência ao desmonte da RAPS e sua continuidade depende da apropriação técnicocientífica e defesa da RAPS pelos trabalhadores e usuários.

Descritores: Educação Permanente; Internato e Residência; Saúde Mental; Serviços de Saúde Mental.

\begin{abstract}
Objective: To understand the historical, political and pedagogical context of the implementation of the Integrated Residency in Collective Mental Health of the School of Public Health of Ceará (RISMC-ESP/CE), and the way the social actors involved in its implementation perceive the reflection of this implementation on the organization of psychosocial care in four municipalities of Ceará, Brazil. Methods: Qualitative, descriptive, exploratory and analytical study, using document analysis, semi-structured interviews and thematic analysis. Results: Four categories have emerged, namely, Historical, political and pedagogical context of the implementation of RISMC-ESP/CE; Continuing interprofessional education in Mental Health (EPISM) in care networks and in the territory; Strengthening of popular participation and anti-asylum fight; and Mental health challenges. Despite the underfunding of SUS and policies that favor the dismantling of the Psychosocial Care Network (RAPS), mental health has been promoted by means of the EPISM, generating intersectoral actions, use of advanced care technologies, and stimulation of social participation in the territory. The main challenges are the social stigma of insanity, the consolidation of care strategies aimed at overcoming the mental institutions and investing in harm reduction, and the stability of health teams for continuity of the implemented actions. Conclusion: RISMC-ESP/CE is a feature to resist RAPS dismantling, and its continuity depends on the technical and scientific appropriation and defense of RAPS by workers and users.
\end{abstract}

Descriptors: Education, Continuing; Internship and Residency; Mental Health; Mental Health Services.

Este artigo foi selecionado, corrigido e aprovado pela Comissão Científica do Fórum Internacional de Sistemas Universais de Saúde, seguindo suas normas e formatação. 


\section{RESUMEN}

Objetivo: Comprender el contexto histórico y politico-pedagogico de la implementación de la Residencia Integrada en Salud Mental Colectiva de la Escuela de Salud Pública de Ceará (RISMC-ESP/CE) y la percepción de los actores sociales involucrados en su implementación sobre sus reflejos en la organización de la atención psicosocial de cuatro municipios de Ceará, Brasil. Métodos: Estudio cualitativo descriptivo, exploratorio y analítico, con análisis documental, entrevistas semiestructuradas y análisis de contenido temático. Resultados: Emergieron cuatro categorías que fueron el Contexto histórico y politico-pedagogico de la implementación de la RISMC-ESP/CE; la Educación permanente interprofesional en Salud Mental (EPISM) de las redes de atención y del territorio; el Fortalecimiento de la participación popular y la lucha contra el manicomio; y los Desafíos de la salud mental. Pese la subfinanciación del SUS y de las políticas que favorecen el término de la Red de Atención Psicosocial (RAPS), hubo la promoción de la salud mental a través de la EPISM generando acciones intersectoriales, la utilización de tecnologías avanzadas de cuidado y el estímulo para la participación social en el territorio. Los principales desafíos son el estigma social de la locura, la consolidación de estrategias de cuidado que superan las instituciones de asilo e invistan en la reducción de daños y la estabilidad de los equipos de salud para el seguimiento de las acciones construidas. Conclusión: La RISMC-ESP/CE es un dispositivo de resistencia para el término de la RAPS y su seguimiento depende de la apropiación técnico-científica y la defensa de la RAPS por trabajadores y usuarios.

Descriptores: Educación Continua; Internado y Residencia; Salud Mental; Servicios de Salud Mental.

\section{INTRODUÇÃO}

Há 30 anos o Brasil viu nascer o Movimento Nacional de Luta Antimanicomial(1), contemporâneo, resultante das lutas pela redemocratização no país, do Movimento de Reforma Sanitária e da Reforma Psiquiátrica, acreditando na viabilidade de uma sociedade mais justa, igualitária e sem manicômios.

Estes movimentos populares e de profissionais pela conquista de direitos de cidadania resultaram na inclusão do Capítulo de Seguridade Social e Saúde na Constituição Cidadã de 1988 e tiveram como consequências, no período de 1988 a 2014, a criação do Sistema Único de Saúde (SUS), os avanços na Reforma Psiquiátrica Brasileira (RPB) e a criação da Rede de Atenção Psicossocial (RAPS), entre tantas outras medidas que significaram a ampliação de direitos sociais.

Apesar dos avanços conquistados, as ameaças de desmonte são constantes. A Emenda Constitucional $n^{\circ}$ 95/2016(2) é apresentada como uma reforma do regime fiscal, mas define o congelamento em 20 anos dos recursos para a saúde pública e políticas sociais em geral, e a redução da participação do Estado na economia. A Portaria do Ministério da Saúde de $n^{\circ} 3.588 / 2017^{(3)}$ reorienta a RAPS, fortalecendo o caráter hospitalocêntrico na saúde mental com o incentivo financeiro aos leitos psiquiátricos.

No contexto da Política Nacional de Educação Permanente em Saúde (PNEPS)(4), emergiram estratégias como os Programas de Residência Multiprofissional (RMS) e Residências Integradas em Saúde (RIS), cursos de educação pelo trabalho que utilizam a educação permanente interprofissional em saúde, metodologias ativas e participativas ${ }^{(5)}$.

A RMS caracteriza-se como programa de pós-graduação "lato sensu" destinado aos graduados em saúde, possuindo em sua metodologia o ensino em serviço, desenvolvido em 60 horas semanais( ${ }^{(6)}$, por dois anos, em regime de dedicação exclusiva. É composta por no mínimo três categorias profissionais da saúde, que compartilham o mesmo processo formativo( ${ }^{(7)}$. Parte-se da premissa de que RMS tem potencial para proporcionar transformações no cotidiano dos serviços de saúde a partir do trabalho colaborativo ${ }^{(5,8,9)}$.

Na saúde mental, tem-se a otimização da atenção psicossocial a partir dessa prática colaborativa ${ }^{(10)}$. No Estado do Ceará, em maio de 2013, iniciou-se o processo de interiorização da Educação Permanente Interprofissional em Saúde por meio da Residência Integrada em Saúde da Escola de Saúde Pública do Ceará (RIS/ESP-CE), tendo como uma das suas ênfases a Saúde Mental Coletiva (RISMC-ESP/CE).

O objetivo do presente estudo foi compreender o contexto histórico e político-pedagógico da implementação da RISMC-ESP/CE, e a percepção dos atores sociais envolvidos na implementação desta sobre os seus reflexos na organização da atenção psicossocial em quatro municípios do Ceará, Brasil.

\section{MÉTODOS}

A pesquisa apresentada é um estudo qualitativo descritivo, exploratório e analítico ${ }^{(11,13)}$. O contexto da pesquisa foi formado pelos quatro municípios cearenses que receberam e concluíram a primeira e a segunda turma da 
RISMC-ESP/CE, sendo iniciadas nos anos de 2013 e de 2014. Nestas turmas foram ofertadas 53 vagas cada, para as categorias de Educação Física, Enfermagem, Psicologia, Serviço Social e Terapia Ocupacional, totalizando 106 vagas ofertadas e 94 profissionais egressos, especialistas em saúde mental coletiva.

$O$ desenho do coletivo de participantes foi definido de forma intencional a fim de garantir a representatividade e a qualidade das informações, sendo o fechamento dado pela redundância ou saturação das mesmas. Assim, os atores sociais do estudo foram selecionados considerando sua importância no processo de implementação da RISMC-ESP/CE a partir das etapas envolvidas, ou seja, desde o planejamento até a certificação da formação dos profissionais egressos (Tabela I).

Tabela I - Identificação e quantificação dos(as) participantes da pesquisa.

\begin{tabular}{lc}
\hline Identificação do(a) Participante & Quantidade \\
\hline Secretário(a) Municipal de Saúde & 4 \\
Coordenador(a) de Saúde Mental & 3 \\
Articulador(a) da RISMC-ESP/CE & 3 \\
Gerentes dos CAPS & 3 \\
Preceptores(as) de Campo & 3 \\
Profissionais dos CAPS & 3 \\
Profissionais Residentes & 15 \\
Total de participantes & 34 \\
\hline
\end{tabular}

Os profissionais do serviço que não atuaram com os residentes da primeira e da segunda turma da RISMC-ESP/ CE e os residentes que não concluíram a RISMC-ESP/CE foram excluídos da amostra.

Como técnicas de coleta de dados foram utilizadas análise documental e entrevistas semiestruturadas. A gravação das entrevistas ocorreu mediante autorização prévia dos participantes e estas foram compostas por perguntas semiabertas ${ }^{(13)}$, possibilitando a construção das informações pertinentes ao estudo.

A análise documental contribuiu para a apreensão dos fatos que permearam a implementação da RISMC-ESP/ $\mathrm{CE}$, sendo composta pela análise dos seguintes documentos: legislação brasileira pertinente à temática trabalhada, editais de seleção dos profissionais residentes, do Regimento Interno, do Projeto Político Pedagógico (PPP) da RIS-ESP/CE, dos bancos de dados de cadastros de preceptores e residentes ${ }^{(2-4,5,7,14-17)}$.

Como técnica de análise, utilizou-se a Análise de Conteúdo Temática ${ }^{(13,18)}$. A análise dos resultados ancorou-se nos referenciais teóricos da RPB e da Atenção Psicossocial ${ }^{(15,16,19,20-25)}$, da Educação Permanente em Saúde ${ }^{(26, " 27)} \mathrm{e}$ da Colaboração Interprofissional| ${ }^{(8,9,10,28)}$, à luz do atual contexto da saúde pública brasileira.

O trabalho de campo realizou-se de julho de 2015 a agosto de 2016, sendo os objetivos da pesquisa explicados de forma clara aos entrevistados, apresentando o Termo de Consentimento Livre e Esclarecido para autorização em participar voluntariamente do estudo, garantindo o sigilo de suas identidades.

A pesquisa seguiu os preceitos éticos da Resolução $n^{\circ}$ 466/12 do Conselho Nacional de Saúde, sendo aprovada pelo Comitê de Ética em Pesquisa da Universidade Federal do Ceará (UFC) com o Parecer nº 1. 121.574.

\section{RESULTADOS E DISCUSSÃO}

Foram reveladas quatro categorias de análise, a saber: Contexto histórico e político-pedagógico da implementação da RISMC-ESP/CE; Educação Interprofissional pelo trabalho em Saúde Mental nas redes de atenção e no território; Fortalecimento da participação popular e da luta antimanicomial; e Desafios da atenção em saúde mental.

\section{Contexto histórico, político-pedagógico da implementação da RISMC-ESP/CE}

A implementação da RISMC-ESP/CE demandou densa articulação interfederativa, interinstitucional e interiorizada. União, Estado e municípios assumiram competências institucionais para o desenvolvimento do Programa (Quadro I). Os 30 municípios interessados em implementá-la, celebraram convênio junto à ESP-CE, que por sua vez, é vinculada aos Ministérios da Saúde e Educação através de editais nacionais de financiamento e autorização da formação na modalidade Residência. 
Quadro I - Competências institucionais dos entes federados envolvidos na implementação da RISMC-ESP/CE.

\begin{tabular}{|c|c|}
\hline \multicolumn{2}{|c|}{ Competências institucionais dos entes federados envolvidos na implementação da RISMC-ESP/CE } \\
\hline Ministério da Saúde & $\begin{array}{l}\text { - Autorização nacional para abertura e implementação da formação na } \\
\text { modalidade residência em saúde } \\
\text { - Financiamento das bolsas de Educação pelo Trabalho para todos os profissionais } \\
\text { residentes; }\end{array}$ \\
\hline Ministério da Educação & $\begin{array}{l}\text { - Autorização nacional para abertura e implementação da formação na modalidade } \\
\text { residência em saúde; } \\
\text { - Legislação (Regulamentação normativa) e } \\
\text { Monitoramento do Programa; } \\
\text { - Coordenação da Comissão Nacional de Residências em Saúde / CNRMS. }\end{array}$ \\
\hline $\begin{array}{l}\text { Governo do Estado: } \\
\text { ESP/CE }\end{array}$ & $\begin{array}{l}\text { - Condução Político-Pedagógica, por meio do corpo docente-estruturante } \\
\text { (tutores): } \\
\text { - Habilitação e Matriciamento Politico-Pedagógico, } \\
\text { permanente e longitudinal, do corpo docente assistencial } \\
\text { (preceptores); } \\
\text { - Docência das atividades teóricas; } \\
\text { - Elaboração de material didático teórico, teórico-prático e prático para residentes } \\
\text { e preceptores; } \\
\text { - Avaliação das atividades-produto resultantes de cada módulo de ensino- } \\
\text { aprendizagem; } \\
\text { - Orientação em pesquisa dos trabalhos de conclusão da residência (TCR); } \\
\text { - Certificação dos concludentes; } \\
\text { - Coordenação da Comissão de Residência } \\
\text { Uni/Multiprofissional em Saúde (COREMU); } \\
\text { - Monitoramento da vinculação das equipes de residentes aos serviços municipais. }\end{array}$ \\
\hline Municípios & $\begin{array}{l}\text { - Seleção e valorização social do corpo de preceptores; } \\
\text { - Implementação das atividades práticas e teórico-práticas; por meio da } \\
\text { preceptoria, matriciada pela da tutoria ESP/CE; } \\
\text { - Vinculação das equipes de residentes aos serviços municipais. }\end{array}$ \\
\hline
\end{tabular}

(Fonte: elaborado pelos autores)

Os fatores de influência da construção desse programa de RMS no Ceará não se limitaram ao contexto estadual, também ampliaram o olhar para a história da reforma sanitária brasileira, da RPB, da criação do SUS e da construção da PNEPS, demonstrando a importância do diálogo no trabalho entre as redes de atenção para o desenvolvimento e aperfeiçoamento do cuidado universal e integral em saúde.

Os dispositivos da RAPS como Centros de Atenção Psicossocial (CAPS), Residências Terapêuticas, Centros de Convivência de Arte e Cultura, Serviços de Urgência Psiquiátrica, Leitos Psiquiátricos em Hospitais Gerais, Equipes de Saúde Mental na Atenção Primária à Saúde (APS), Atenção à Saúde Mental na Estratégia Saúde da Família, foram resultantes do processo histórico da Reforma Sanitária e da RPB ${ }^{(15,16)}$, permitindo o acesso a tecnologias psicossociais de cuidado tais como gestão da clínica, acolhimento, matriciamento e clínica peripatética a milhões de brasileiros.

Pode-se considerar que a construção da RAPS no Ceará iniciou a partir de sua regulamentação com a Lei Mário Mamede, Lei Estadual no 12.151/1993(29), pois esta é anterior a Lei Federal 10.216/2001 ${ }^{(17)}$, e desde então dispôs sobre a extinção progressiva de unidades psiquiátricas hospitalares por outros mecanismos assistenciais que não isolassem as pessoas em sofrimento psíquico do convívio social e familiar. Assim, a referida Lei apresentou o prazo de cinco anos para a extinção de leitos psiquiátricos e a implantação dos serviços substitutivos, proibindo ainda a construção e a ampliação de hospitais psiquiátricos independentemente de serem públicos ou privados.

No cenário nacional, apesar da Lei Federal 10.216/2001(17), ainda em vigor, ter assegurado os direitos à proteção das pessoas acometidas de transtorno mental, sem qualquer forma de discriminação quanto à raça, cor, sexo, orientação sexual, religião, opção política, nacionalidade, idade, família, recursos econômicos e ao grau de gravidade ou tempo de evolução de seu transtorno, na prática, esses direitos estão gravemente ameaçados pela portaria do Ministério da Saúde de $n^{\circ} 3.588$, de 21 de dezembro de $2017^{(3)}$. 
A citada portaria permite novamente que enfermarias com até "30 pacientes" sejam criadas em hospitais psiquiátricos, além de prever internamentos de longa duração, dando lugar ao amontoamento de brasileiros com agravos em saúde mental em situação de iniquidade.

Em comparação, o padrão de atenção em saúde mental ofertado a associados de planos de saúde no Brasil, estabelece por meio da Resolução Normativa da Agência Nacional de Saúde (ANS) n. 428/2017(30), em seu artigo 10 , que "a atenção prestada aos portadores de transtornos mentais deverá priorizar o atendimento ambulatorial e em consultórios, utilizando a internação psiquiátrica apenas como último recurso terapêutico e sempre que houver indicação do médico assistente".

A Política de Austeridade econômica executada no Brasil por meio do congelamento dos recursos para a saúde, educação e área social durante 20 anos através da Emenda Constitucional $n^{\circ}$ 95/2016 (2), se for mantida, poderá reduzir o orçamento para políticas sociais e refletirá no desmonte de serviços de saúde, inclusive os de atenção psicossocial, deixando milhões de cidadãos sem acesso à atenção integral em saúde.

O corte de despesas em um período de recessão econômica é considerado um desastre financeiro e humano, ou seja, colocar em risco à saúde da população não é uma escolha econômica inteligente. Evidências de estudos internacionais comprovam a piora dos indicadores da saúde através da redução com os gastos sociais ${ }^{(26)}$

Dentro desse contexto, a RISMC-ESP/CE objetiva formar especialistas nessa área com base nos pressupostos da luta antimanicomial, da RPB e do SUS, com ênfase na Política Nacional de Saúde Mental, considerando a indissociabilidade entre educação, trabalho e cidadania ${ }^{\left({ }^{31}\right)}$. A partir disto, é perceptível a importância da sua implementação para o SUS e para a atenção psicossocial.

\section{Educação Interprofissional pelo trabalho em Saúde Mental nas redes de atenção e no território}

O PPP da RIS/ESP-CE ${ }^{(31)}$ foi criado com a previsão do desenvolvimento de competências comuns e competências específicas para cada uma das onze ênfases constituintes, dentre elas a RISMC-ESP/CE, sendo suas características gerais: o currículo baseado em competências; o processo de ensino-aprendizagem pautado pelas necessidades populacionais; processo de trabalho resolutivo; o desenvolvimento de competências profissionais para o Campo (competências comuns) e Núcleo (competências específicas de cada categoria) de atuação profissional no SUS; o processo de ensino-aprendizagem pautado na ação-reflexão-ação (Práxis) e na prática colaborativa interprofissional; a atuação docente Freiriana em ato; a atuação-formativa implicada com o SUS (ato em saúde como ato formativo e ato político).

No PPP da RIS-ESP/CE ${ }^{(31)}$ identificou-se o módulo "Território e Saúde" como disparador da imersão dos residentes nos locais de atuação, sendo este um módulo transversal comum à todas as profissões, ênfases da RIS-ESP/CE e cenários de atuação ${ }^{(32)}$. As ações de mapeamento do território vivo ${ }^{(33)}$ ocorrem com o planejamento da sequência de ações e arranjos territoriais mais favoráveis a um rendimento máximo das metas traçadas, tendo um papel fundamental na organização da vida coletiva e individual das pessoas.

As ações com base territorial foram desenvolvidas pelos residentes, ultrapassando os muros dos CAPS, o que foi denominado nas falas por "sair do CAPS, fora do CAPS, extra CAPS", coerentemente com os pressupostos da RPB e da atenção psicossocial em saúde ${ }^{(19)}$, caminhando no desenvolvimento da colaboração interprofissional(8).

Compreendeu-se o território como um espaço vivo, repleto de possibilidades de atuação para responder às necessidades de saúde da comunidade. Os participantes da pesquisa ressaltaram a relevância e o contínuo desafio da atuação com base no território orientando processos de desinstitucionalização das pessoas com agravos em saúde mental:

"(Os residentes) vêm com esse gás de estar mais no território, de cumprir realmente o que é saúde mental na expectativa da Reforma Psiquiátrica Brasileira, extra muro, extra CAPS. Então, isso é um dos pontos que eu considero assim [...] muito bom, (os residentes) dão essa alavancada, esse gás [...] de estar fazendo essas articulações no território e no desenvolvimento das (ações) intersetoriais." (Ger2)

As características apontadas podem ser exemplificas pelas competências profissionais transversais desenvolvidas para o perfil do egresso por meio do PPP do Programa ${ }^{(31,34)}$. As competências transversais são aquelas consideradas comuns a todas as categorias profissionais e de todas as especialidades atuantes no SUS e, portanto, esperadas nos egressos de todas as profissões e ênfases da RIS-ESP/CE. (Quadro II). 
Quadro II - Competências profissionais transversais constituintes do Projeto Político Pedagógico da Residência Integrada em Saúde do Ceará.

\begin{tabular}{|c|l|}
\hline \multicolumn{2}{|l}{$\begin{array}{l}\text { Competências profissionais transversais constituintes do Projeto Político Pedagógico da Residência Integrada em Saúde } \\
\text { do Ceará }\end{array}$} \\
\hline 1 & Desenvolvimento do processo de trabalho em saúde a partir do território. \\
\hline 2 & $\begin{array}{l}\text { Atuação no SUS com fortalecimento da atenção integral nas redes de atenção, nos serviços de saúde e nas } \\
\text { ações junto aos usuários e coletivos. }\end{array}$ \\
\hline 3 & $\begin{array}{l}\text { Atuação no SUS a partir da intersetorialidade, fortalecendo a articulação entre demais políticas públicas e } \\
\text { dispositivos comunitários. }\end{array}$ \\
\hline 4 & $\begin{array}{l}\text { Atuação no SUS com o fortalecimento da colaboração interprofissional e do trabalho em equipe nos serviços } \\
\text { de saúde. }\end{array}$ \\
\hline 5 & $\begin{array}{l}\text { Atuação no SUS com compromisso ético político com a reforma sanitária e psiquiátrica, e seus atores (residentes, } \\
\text { preceptores, gestores, demais profissionais e usuários). }\end{array}$ \\
\hline 6 & $\begin{array}{l}\text { Desenvolvimento de processos de educação permanente no SUS comprometidos com a transformação do } \\
\text { cenário de prática. }\end{array}$ \\
\hline 7 & $\begin{array}{l}\text { Produção e criação do conhecimento com compromisso com a transformação da realidade e emancipação dos } \\
\text { sujeitos envolvidos. }\end{array}$ \\
\hline 8 & Desenvolvimento de ações para promoção da saúde e defesa da vida \\
\hline 9 & Atuação no SUS embasada na educação popular em saúde. \\
\hline 10 & Articulação comunitária e fortalecimento da participação e do controle social. \\
\hline
\end{tabular}

(Fonte: elaborado pelos autores)

A saúde mental movimenta saberes e práticas transdisciplinares, buscando olhares das outras políticas sociais e do saber popular. A atuação no território, possibilitando a articulação tanto com os equipamentos da saúde, quanto com os de outras políticas sociais fomentam a atuação intersetorial e a desinstitucionalização(19,20):

"A residência tem essa característica de fazer com que vários dispositivos que o município tem se comuniquem.

[...] Isso aí fortalece muito o cuidado." (Res9)

O conceito e as dimensões da intersetorialidade foram discutidos nas atividades teóricas da RISMC-ESP/CE enquanto as práticas correspondentes ocorreram cotidianamente nos serviços e na comunidade, fortalecendo a atuação profissional e a RAPS:

"[...] A gente conseguiu fazer algumas alianças legais, algumas parcerias, principalmente com a rede intersetorial, isso foi muito rico na assistência social, foi quando deu para perceber que a saúde mental não é só saúde em termos biológicos, mas também é cultura, é política, é parte econômica e tudo isso está envolvido." (Res11)

O processo formativo de saúde mental desenvolvido demonstra que a RISMC-ESP/CE fomenta e implementa Educação Permanente em Saúde (EPS) pautada no trabalho em rede e para tanto, utiliza de atividades e estratégias pedagógicas indutoras da intersetorialidade para o alcance da integralidade. Nesse sentido, constata-se a vivência da Clínica Peripatética ${ }^{(25)}$, haja vista a itinerância e busca permanente pela realização de atividades nos espaços de maior identidade social-territorial dos usuários envolvidos e constituindo, de forma permanente, pontos de encontro nos municípios e regiões de atuação-formativa.

A atuação da RISMC-ESP/CE é orientada pelo trabalho a partir das redes de atenção à saúde e da articulação intersetorial a partir da integralidade do cuidado - percepções encontradas no discurso dos atores sociais da pesquisa. A integralidade está relacionada à noção de continuidade de cuidados implicando que os serviços sejam abrangentes ${ }^{(21)}$, podendo substituir funções antes limitadas ao aparato hospitalocêntrico.

O PPP do Programa objetiva o desenvolvimento de competências para a implementação de atividades na perspectiva da integralidade (Rede e Sujeito): consultas conjuntas, matriciamento em saúde mental na APS, grupos terapêuticos, grupos de educação em saúde com diversos públicos (como crianças, adolescentes, mulheres, idosos, homens, população LGBT e população em situação de rua), visitas domiciliares, discussão de casos, relações e práticas intrasetoriais, intersetoriais e interorganizacionais entre os dispositivos, atendimentos descentralizados (nos espaços do território), rodas de educação permanente e demais atividades planejadas de acordo com as demandas identificadas. 
O desenvolvimento do processo de territorialização e da atuação intersetorial potencializou o diálogo entre APS e a RAPS. Essas estratégias de atuação potencializaram o cuidado psicossocial de base comunitária, buscando construir uma atuação em saúde mental orientada para necessidades sociais, pressuposto da atenção psicossocial(20,22).

Outra dimensão significativa que emergiu na atual pesquisa foi a construção de ferramentas de cuidado no âmbito da gestão da clínica: acolhimento, clínica ampliada, matriciamento e projeto terapêutico singular. Essa inovação contribuiu para a modificação do modelo de atenção em saúde mental, passando a abordar os usuários integralmente, reconhecendo suas várias dimensões e não apenas o seu contexto psíquico, além de potencializarem a colaboração interprofissional no processo de ensino e trabalho ${ }^{(8)}$ :

"Tanto na questão do vínculo, por elas (residentes) estarem dentro do serviço participando das ações, dos serviços e do fluxo do serviço. Elas (residentes) participam sobre tudo na questão da escuta, do vínculo e do acolhimento. [...] Se existe escuta, acolhimento e vínculo vai existir a integralidade das ações, uma vez que elas estão ligadas também ao território, às outras instituições, às outras políticas públicas.” (Ger2)

Nas experiências de RISMC/ESP/CE o apoio matricial foi incorporado como estratégia de organização do processo de trabalho, de educação permanente e de cuidado em saúde mental. Os participantes da pesquisa explicam o apoio matricial como uma inovação que potencializa a colaboração interprofissional ${ }^{(8)}$ :

"Matriciamento, eu acho que (é) uma contribuição para nossos serviços. O conhecimento também porque você acaba levando o seu conhecimento em alguns lugares e acaba que também se fazendo conhecer [...]." (Ger3)

Outro avanço identificado refere-se à colaboração interprofissional realizada por meio da RISMC-ESP/CE. Nesta são formados profissionais de cinco diferentes categorias profissionais (Educação Física, Enfermagem, Psicologia, Serviço Social e Terapia Ocupacional) na especialidade saúde mental coletiva, ou seja, com competências comuns para este campo de atuação, nesse caso o SUS, RPB, saúde mental e atenção psicossocial.

A partir dessa percepção, cabe ressaltar que a colaboração interprofissional aperfeiçoa essa interação entre os profissionais das equipes melhoram a atenção à saúde dos usuários:

"[...] Os profissionais viram que eles tinham que se mexer e cada um tinha que sair da sua casinha. [...] Ser tanto multi como interprofissional e isso mexeu com cada um, mexeu com o dinamismo do contexto do profissional e isso foi impactante." (Res8)

A colaboração interprofissional( ${ }^{(8)}$ ocorre pois mais de duas categorias profissionais da saúde atuam e aprendem dentro das realidades dos serviços. Logo, "[...] é importante essa questão da equipe multiprofissional nessa perspectiva de todos falarem a mesma coisa, de entender os sintomas, as causas e de poderem atuar juntos" (Res4). Essa importância ressaltada aparece nos discursos dos atores sociais, focando nas mudanças de visão sobre a prática profissional, mostrando que esta pode e deve ser compartilhada e não solitária, ou seja, não deve ser realizada de forma fragmentada por cada categoria profissional.

Desse modo, a RISMC-ESP/CE disparou avanços na organização na atenção psicossocial nos municípios estudados e fizeram com que a RAPS, através da atuação interprofissional, fosse fortalecida mesmo diante dos limites impostos pela realidade do subfinanciamento do SUS nos municípios e a falta de investimentos na RAPS ${ }^{(1,35,36,37,38)}$.

\section{Fortalecimento da participação popular e da luta antimanicomial}

Surgiu, através da análise, a dimensão da participação social incentivada pela RISMC-ESP/CE, sendo possível identificar ações inspiradas na luta pelo direito à saúde e na luta antimanicomial. As Conferências Nacionais de Saúde Mental realizadas desde 1987 são exemplos das possibilidades da inserção dos atores sociais nas discussões e construções das políticas de Saúde Mental e atenção psicossocial|(19).

Os movimentos sociais até 2014 conseguiram lutar e aprovar a legislação que determina a substituição dos leitos psiquiátricos por uma rede integrada de Atenção à Saúde Mental. No entanto, ao se falar em cidadania e direitos não basta apenas aprovar leis, sabendo que o campo da Saúde Mental e atenção psicossocial é um processo social complexo, necessitando mudar mentalidades, atitudes e relações sociais ${ }^{(19)}$. Diante disto, tem-se os retrocessos impostos pelo atual governo brasileiro, não considerando o repúdio a tais medidas por militantes da luta antimanicomial( ${ }^{1,35,36,37,38)}$.

A partir da implementação da RISMC-ESP/CE, a participação social passou a existir nos territórios de atuação dos residentes, como também em assembleias de usuários e reuniões de conselhos de saúde. Pré-conferências e conferências de saúde entraram no calendário dos usuários e de seus familiares. 
"[...] Outra inovação diz respeito ao olhar sobre a saúde mental, principalmente no CAPS AD que nasceu com a residência, é a participação dos usuários, por exemplo, a assembleia de usuários e familiares. É uma prática mensal que está no calendário e que tem ampla participação. [...]." (Prec2)

A RISMC-ESP/CE a partir da participação social potencializou a reflexão sobre a construção de uma sociedade sem manicômios ${ }^{(23)}$, atuando em estratégias de desinstitucionalização da loucura no cotidiano da vida social. O que antes 'era lugar para doido' passou a ser utilizado pela comunidade.

O modelo de atenção em saúde mental adotado merece destaque, mas apesar do desenvolvimento da atenção psicossocial e do comprometimento com as lutas antimanicomiais, foram perceptíveis nos municípios a cultura das práticas ambulatoriais e hospitalocêntricas, exigindo dos residentes a execução de ações condizentes com a RPB. O estigma da loucura e as formas hegemônicas de lidar com a mesma ${ }^{(23)}$ ainda existem na sociedade, necessitando do comprometimento dos profissionais de saúde para a desconstrução dos olhares estigmatizantes.

\title{
Os desafios da atenção em saúde mental
}

É possivel compreender a RISMC-ESP/CE como promotora de saúde mental nos territórios por onde foi desenvolvida, porém ainda existem desafios sobre a compreensão e aceitação das pessoas com agravos em saúde mental nas comunidades, exigindo dispositivos coerentes com os pressupostos da RPB e da Atenção Psicossocial(19,20):

\begin{abstract}
"Estigma, o preconceito, o medo do louco, as pessoas ainda têm aquele medo do louco. [...] Chega um paciente de saúde mental, por exemplo, (na APS) e todo mundo comentando, porque ele vai quebrar tudo, porque ela vai me agredir e a gente sempre tenta voltar essa questão da humanização do cuidado, assim como qualquer outro paciente ele também tem que ser cuidado. [...] Não é do CAPS, o paciente é do território. [...]." (Res2)
\end{abstract}

Os atores sociais da RISMC-ESP/CE tentaram desmistificar os estigmas sobre os agravos em saúde mental, mas a luta é cotidiana e a atuação de profissionais de saúde comprometidos com a RPB otimiza essa construção, lembrando-se também da necessidade de não focar a atuação apenas nas instituições. O 'sair do muro do CAPS' remete à construção de condições, situações e organizações dispostas a darem respostas às necessidades, podendo acontecer através da construção de espaços e projetos coletivos capazes de intervir na realidade, pois a ideia de cuidado e liberdade são pressupostos da vida e do desenvolvimento da cidadania ${ }^{(24)}$.

Na RISMC-ESP/CE o PPP prevê o rodízio exclusivamente em serviços abertos da RAPS e não autoriza qualquer vivência, enquanto profissional-residente, em instituições de caráter asilar. Não são realizados rodízios de atuaçãoformativa em hospital psiquiátrico, manicômios judiciários e comunidades terapêuticas. As estratégias de cuidado contemplam práticas de consultório e território, individuais e coletivas, institucionais e comunitárias e no escopo da Integralidade do Cuidado (Vivências práticas de Prevenção, Atenção, Promoção e Reabilitação em Saúde Mental).

Os partícipes do cuidado proposto pela RISMC-ESP/CE constituem as Redes Vivas (com suas dimensões Institucional e Afetiva) forjadas pela imersão radical dos profissionais-residentes nos territórios de atuação-formativa e nos contextos sócio-culturais dos usuários, famílias e comunidades de interação nos dois anos de curso da residência, fomentando a defesa da vida, a participação popular e o cuidado, não a mera abordagem do agravo. $O$ cuidado às pessoas em uso problemático de álcool ou outras drogas, por exemplo, adota a estratégia de redução de danos, como estratégia de cuidado integral.

E, por fim, as pessoas em sofrimento psíquico necessitam de um cuidado longitudinal, sendo desejável a estabilidade das equipes de saúde, rara na realidade brasileira. A continuidade está estritamente relacionada ao acesso aos serviços e ao cuidado( ${ }^{(21)}$, desse modo, na RISMC-ESP/CE em seus dois anos de duração, a formação profissional e institucional para a atenção psicossocial cria uma continuidade mínima, porém insuficiente, no cuidado aos usuários.

\section{CONSIDERAÇÕES FINAIS}

As características apresentadas no PPP da RIS-ESP/CE implementadas no SUS dos municípios e constatadas na pesquisa de campo deste estudo, situam o Programa como um dispositivo de Educação Permanente Interprofissional em Saúde Mental que ativa profissionais de saúde para a luta antimanicomial, fortalece a RPB e que resiste ao desmonte da rede de saúde mental.

Esta resistência é mais ou menos consistente a depender dos valores e posicionamentos político-ideológicos das gestões municipais, da apropriação técnico-científica e política da luta antimanicomial e RPB pelos trabalhadores e usuários do SUS, o que se reflete no controle social em saúde nos municípios de vivência da RISMC-ESP/CE. 
Os participantes da pesquisa perceberam a implementação da RISMC-ESP/CE como potencializadora da ampliação das práticas de cuidado, com o desenvolvimento de estratégias e tecnologias de abordagem comunitária, dialogando com o cotidiano da vida social. Na compreensão dos autores, a RAPS no Ceará tem sido fortalecida e até mesmo estruturada através das inovações promovidas pela RISMC-ESP/CE, resultando na ampliação do acesso à atenção psicossocial, ao acolhimento, à articulação dos pontos de atenção e das redes de saúde no território e a vinculação de usuários à estes, promovendo a saúde mental. Como reflexos positivos destacam-se inovações de práticas em saúde mental, processo terapêutico de base territorial, educação permanente em saúde mental, trabalho em equipe e colaboração interprofissional, mudanças na vida social dos usuários e de seus familiares com a participação social, acolhimento, clínica ampliada e projeto terapêutico singular, resultando na construção de vínculos, desmistificação de estigmas sobre a loucura e sobre a atenção em saúde mental, e formação de profissionais comprometidos com as questões da Reforma Sanitária e da RPB.

Os desafios incluem estigmas da população, de profissionais de saúde e de outras políticas públicas sobre a loucura e à atenção em saúde mental; consolidação de estratégias de cuidado que superem as instituições de caráter asilar e promovam a redução de danos para pessoas em uso problemático de álcool e outras drogas, e estabilidade das equipes de saúde para a continuidade das ações construídas a partir da RISMC-ESP/CE para o cuidado contínuo e integral aos usuários.

Contudo, a implementação da RISMC-ESP/CE demonstra a real possibilidade de se promover saúde mental de base territorial e antimanicomial dentro das redes de atenção à saúde. Sugere-se a pactuação coletiva e solidária dos atores e das instituições partícipes do SUS, pois mesmo diante dos avanços encontrados no contexto da atenção psicossocial, da RPB, da resistência ao desmonte da RAPS e da Educação Permanente Interprofissional em Saúde Mental, se faz necessário o enfrentamento dos desafios aqui registrados na implementação da RISMCESP/CE de forma interiorizada.

\section{AGRADECIMENTOS}

Ao Ministério da Saúde e à Coordenação de Aperfeiçoamento de Pessoal de Nível Superior (CAPES). Aos professores Prof. Dr. Erasmo Ruiz, Profa. Dra. Ângela Linhares e Profa. Dra. Ana Ecilda Ellery. À Rúbia Andrade. Aos atores sociais da Residência Integrada em Saúde Mental Coletiva da Escola de Saúde Pública do Ceará.

\section{CONFLITOS DE INTERESSE}

O manuscrito não apresenta relações que possam implicar em potencial conflitos de interesse.

O artigo é fruto da dissertação de mestrado intitulada "Os reflexos da implementação da Residência Integrada em Saúde Mental Coletiva do Ceará na Atenção Psicossocial" (141 páginas) apresentada ao Programa de Pósgraduação em Saúde Coletiva do Departamento de Saúde Comunitária da Universidade Federal do Ceará em 2017.

Fonte de Financiamento: Coordenação de Aperfeiçoamento de Pessoal de Nível Superior (CAPES) e Laboratório de Pesquisas em Ensino e Gestão do Conhecimento, da Educação e do Trabalho na Saúde - Edital Pró-Ensino na Saúde 24/2010. Processo n² 23038.009824/2010-01.

\section{REFERENCIAS}

1. Movimento Nacional da Luta Antimanicomial. Nota de repúdio contra o desmonte da Política de Saúde Mental [Internet]. 2017 [acesso em 18 jan 2018]. Disponível em: http://www.cressrj.org.br/site/wp-content/uploads/2017/12/ NOTA_DE_REPUDIO_CONTRA_O_DESMONTE_DA_SA\%C3\%9ADE_MENTAL_MNLA.pdf.

2. Brasil. Presidência da República. Emenda Constitucional n. 95, de 15 de dezembro de 2016. Diário Oficial da República Federativa do Brasil, Brasília. 2016. [acesso em 2018 Jan 12]. Disponível em: https://www.jusbrasil. com.br/diarios/133607114/dou-secao-1-16-12-2016-pg-2.

3. Brasil. Ministério da Saúde. Portaria n. 3588, de 21 de dezembro de 2017. Diário Oficial da República Federativa do Brasil, Brasília. 2017. [acesso em 2018 Jan 12]. Disponível em: http://www.brasilsus.com.br/index.php/ legislacoes/gabinete-do-ministro/17904-portaria-n-3-588-de-21-de-dezembro-de-2017.

4. Brasil. Ministério da Saúde. Portaria n. 198, de 13 de fevereiro de 2004. Institui a Política Nacional de Educação Permanente em Saúde como estratégia do Sistema Único de Saúde para a formação e o desenvolvimento de trabalhadores para o setor e dá outras providências. Brasília: Ministério da Saúde; 2004. 
5. Brasil. Ministério da Saúde. Residência Multiprofissional em Saúde: experiências, avanços e desafios [Internet]. Brasília: Ministério da Saúde; 2006. [acesso em 2015 Ago 2015]. Disponível em: http://bvsms.saude.gov.br/bvs/ publicacoes/residencia_multiprofissional.pdf.

6. Brasil. Ministério da Saúde, Ministério da Educação. Portaria Interministerial n. 45, de 12 de janeiro de 2007. Dispõe sobre a Residência Multiprofissional em Saúde e a Residência em Área Profissional da Saúde e institui a Comissão Nacional de Residência Multiprofissional em Saúde. Brasília: Ministério da Saúde, Ministério da Educação; 2007.

7. Brasil. Ministério da Saúde, Secretaria de Educação Superior, Comissão Nacional de Residência Multiprofissional em Saúde. Resolução CNRMS n. 2, de 13 de abril de 2012. Diário Oficial da República Federativa do Brasil, Brasília. 2012.

8. Soares GM. Colaboração e educação interprofissional na pós-graduação em saúde: estudo de caso da residência multiprofissional em saúde da família [dissertação]. Fortaleza (CE): Universidade Federal do Ceará, Faculdade de Medicina; 2015.

9. Ellery AEL. Interprofissionalidade na estratégia saúde da família: condições de possibilidade para a integração de saberes e a colaboração interprofissional [tese]. Fortaleza (CE): Universidade Federal do Ceará, Faculdade de Medicina; 2012.

10. Organização Mundial da Saúde. Marco para Ação em Educação Interprofissional e Prática Colaborativa. Genebra (CH): OMS, Departamento de Recursos Humanos para a Saúde; 2010.

11. Creswell JW. Projeto de Pesquisa: métodos qualitativo, quantitativo e misto. Tradução Magda Lopes. 3a ed. Porto Alegre: Artmed; 2010.

12. Denzin KN, Giardina MD, editores. Qualitative Inquiry Through a critical lens. New York: Routledge; 2016.

13. Minayo MCS. O desafio do conhecimento: pesquisa qualitativa em saúde. 14a ed. São Paulo: Hucitec; 2014.

14. Brasil. Secretaria de Atenção à Saúde, Departamento de Ações Programáticas Estratégicas. Saúde mental no SUS: os centros de atenção psicossocial. Brasília: Ministério da Saúde; 2004.

15. Brasil. Portaria n. 3088, de 23 de dezembro de 2011. Institui a Rede de Atenção Psicossocial para pessoas com sofrimento ou transtorno mental e com necessidades decorrentes do uso de crack, álcool e outras drogas, no âmbito do Sistema Único de Saúde. Diário Oficial da República Federativa do Brasil, Brasília. 2011.

16. Brasil. Ministério da Saúde. Guia prático de matriciamento em saúde mental. Brasília (DF): Ministério da Saúde; 2011.

17. Brasil. Lei n. 10.216, de 06 de abril de 2001. Diário Oficial da República Federativa do Brasil, Brasília. 2001. [acesso em 2015 Ago 25]. Disponível em: http://www.planalto.gov.br/ccivil_03/LEIS/LEIS_2001/L10216.htm.

18. Bardin L. Análise de conteúdo. Lisboa: Edições 70; 2009.

19. Amarante P. Saúde mental e atenção psicossocial. Rio de Janeiro (RJ): Editora FIOCRUZ; 2007. (Coleção Temas em Saúde).

20. Yasui S, Costa-Rosa A. A estratégia atenção psicossocial: desafio na prática dos novos dispositivos de saúde mental. Saúde Debate. 2008;32(78-79-80):27-37.

21. Carvalho MCA. Saúde mental na atenção básica. In: Jorge MAS, Carvalho MCA, Silva PRF, organizadores. Políticas e cuidado em saúde mental: contribuições para a prática profissional. Rio de Janeiro: Editora FIOCRUZ; 2014. p. 59-74.

22. Dimenstein M, Sales AL, Galvão E, Severo AK. Estratégia da atenção psicossocial e a participação da família no cuidado em saúde mental. Physis. 2010;20(4):1209-26.

23. Amarante $\mathrm{P}$, coordenador. Loucos pela vida: a trajetória da reforma psiquiátrica no Brasil. 2a ed. Rio de Janeiro: Editora FIOCRUZ; 1995.

24. Rotelli F. Formação e construção de novas instituições em saúde mental. In: Amarante P, Cruz LB, organizadores. Saúde Mental, formação e crítica. Rio de Janeiro: Laps; 2015. p. 37-50.

25. Lancetti A. Clínica peripatética. São Paulo: Hucitec; 2008. 
26. Stuckler D, Basu S. A economia desumana: porque mata a austeridade. Lisboa: Editorial Bizâncio; 2014.

27. Carvalho YM, Ceccim RB. Formação e educação em saúde: aprendizados com a saúde coletiva. In: Campos GWS, Bonfim JRA, Minayo MCS, Akerman M, Drumont M Jr, Carvalho YM, organizadores. Tratado de Saúde Coletiva. 2a. ed. São Paulo: Hucitec; 2012. p. 137-70.

28. Centro para o Avanço da Educação Interprofissional. Education: The definiton. Fareham (UK): CAIPE; 2002.

29. Ceará. Lei Estadual n. 12.151, de 29 de julho de 1993. Dispõe sobre a extinção progressiva dos hospitais psiquiátricos e sua substituição por outros recursos assistenciais, regulamenta a internação psiquiátrica compulsória, e dá outras providências. Diário Oficial do Estado do Ceará, Fortaleza. 1993.

30. Brasil. Agência Nacional de Saúde Suplementar. Resolução Normativa n. 428, de 7 de novembro de 2017. Brasília: ANS; 2017.

31. Ceará. Secretaria de Saúde do Estado, Escola de Saúde Pública do Ceará. Projeto Político Pedagógico. Fortaleza: ESP/CE; 2012.

32. Ceará. Residência em Saúde do Ceará. Manual pedagógico território e saúde. Fortaleza: ESP/CE; 2017.

33. Santos M. A Natureza do espaço: técnica e tempo, razão e emoção. 4a. ed. São Paulo: Editora da Universidade de São Paulo; 2006.

34. Ceará. Secretaria de Saúde do Estado do Ceará, Escola de Saúde Pública do Ceará. Residência Integrada em Saúde. Regimento RIS-ESP/CE. Fortaleza: ESP/CE; 2013.

35. Associação Brasileira de Saúde Coletiva. Nota Abrasco contra os retrocessos da CGMAD/MS frente à política brasileira de Saúde Mental [Internet]. 2017; [acesso em 2018 Jan 20]. Disponível em: https://www.abrasco.org. $\mathrm{br} / \mathrm{site} /$ noticias/posicionamentos-oficiais/retrocessos-saude-mental-governo-temer/32436/.

36. Conselho Federal de Serviço Social. Mudanças na Política Nacional de Saúde Mental: mais uma ameaça do governo ilegítimo: CFESS divulga nota pública sobre a questão [Internet]. 2017; [acesso em 2018 Jan 13]. Disponível em: http://www.cfess.org.br/visualizar/noticia/cod/1439.

37. Conselho Federal de Psicologia. CFP repudia mudanças na política de saúde mental [Internet]. 2017; [acesso em 2018 Jan 15]. Disponível em: http://site.cfp.org.br/repudio-mudancas-politica-saude-mental/.

38. Por uma sociedade sem manicômios. Carta de Bauru - 30 anos [Internet]. 2017; [acesso em 2018 Jan 15]. Disponível em: http://site.cfp.org.br/wp-content/uploads/2017/12/CARTA-DE-BAURU-30-ANOS.pdf.

\section{Endereço para correspondência:}

Aline Luiza de Paulo Evangelista

Rua Monte Claro, 213

Bairro: Jóquei Clube

CEP: 60510098 - Fortaleza - CE - Brasil

E-mail: alineluizaevangelista@hotmail.com 